PROCEEDINGS OF THE

AMERICAN MATHEMATICAL SOCIETY

Volume 127, Number 10, Pages 2969-2973

S 0002-9939(99)04941-2

Article electronically published on April 28, 1999

\title{
THERE ARE NO DENTING POINTS IN THE UNIT BALL OF $W C(K, X)$
}

\author{
T. S. S. R. K. RAO
}

(Communicated by Palle E. T. Jorgensen)

\begin{abstract}
For an infinite compact set $K$ and for any Banach space $X$ we show that the unit ball of the space of $X$-valued functions that are continuous when $X$ is equipped with the weak topology, has no denting points.
\end{abstract}

\section{INTRODUCTION}

For a compact Hausdorff space $K$ and for any Banach space $X$, let $W C(K, X)$ denote the space of $X$-valued functions on $K$ that are continuous when $X$ has the weak topology, equipped with the supremum norm. A point $x_{0}$ in the unit ball $X_{1}$ of a Banach space $X$ is said to be a denting point if $x_{0}$ is an extreme point and a point of continuity for the identity map $i:\left(X_{1}\right.$, weak $) \rightarrow\left(X_{1},\|\|\right)$. (That this is equivalent to the standard definition of a denting point (see [DU]) is a result from [LLT].) In this note we show that when $K$ is infinite given any $f \in W C(K, X)$ of norm one, there exists a sequence $\left\{f_{n}\right\} \subset W C(K, X)_{1}$ such that $f_{n} \rightarrow f$ in the weak toplogy but not in the norm topology. As a consequence we conclude that $W C(K, X)_{1}$ has no denting points. Our results also extend some results of Grzasślewicz $[\mathrm{G}]$ on the non-availability of strongly exposed points in the unit ball of the space of operator valued continuous functions. For the space of operators we show that for any infinite compact set $K$ and for any $X$ there are no denting points in $\mathcal{L}(X, C(K))_{1}$. Turning to injective tensor products, we show that for any infinite dimensional space $Y$ such that $Y^{*}$ is isometric to an $L^{1}(\mu)$ space (the so-called $L^{1}$ predual spaces; see $[\mathrm{L}]$, Chapter 7 ) and for any $X$, there are no denting points in the unit ball of $Y \otimes_{\epsilon} X$.

\section{MAIN RESULT}

To establish our result, we need the description of the extreme points of $W C(K, X)_{1}^{*}$ given in [De]. Let us recall from [De] that a $\wedge \in W C(K, X)^{*}$ is a point functional if there is a $t_{0} \in K$ so that $\wedge(f a)=f\left(t_{0}\right) \wedge(a)$ for each $f \in C(K)$ and $a \in W C(K, X)$.

Theorem 1 (De Reyna et al.). If $\wedge$ is an extreme point of $W C(K, X)_{1}^{*}$, then $\wedge$ is a point functional (see [R1] for a simpler proof of this result).

Received by the editors September 9, 1996 and, in revised form, April 15, 1997 and January $5,1998$.

1991 Mathematics Subject Classification. Primary 46B20, 46E40.

Key words and phrases. Denting point, vector valued continuous functions.

(C)1999 American Mathematical Society 
Theorem 2. Let $K$ be an infinite compact set. Let $f \in W C(K, X),\|f\|=1$. There exists a sequence $\left\{f_{n}\right\}$ in $W C(K, X)_{1}$ such that $f_{n} \rightarrow f$ in the weak topology but not in the norm topology. Consequently there are no denting points in $W C(K, X)_{1}$.

Proof. Throughout the proof to show the weak convergence of a sequence $\left\{f_{n}\right\}$, we invoke Rainwater's theorem ([D], page 155) and verify the convergence of $\left\{\wedge\left(f_{n}\right)\right\}$ for extreme points $\wedge$ of $W C(K, X)_{1}^{*}$. We now divide the proof into 3 cases.

Case (i). Suppose $f(K)$ is infinite and has a non-zero accumulation point.

Since $f(K)$ is a weakly compact set and since for such sets a result of Eberlein's guarantees that accumulation points can be approximated by sequences (see [D], page 22 and also $[\mathrm{H}]$, page 149), we may assume that there exists a sequence $\left\{t_{n}\right\} \subset K$ such that $f\left(t_{n}\right) \rightarrow x_{0}, x_{0} \neq 0$ in the weak topology. Choose pairwise disjoint open sets $U_{n}$ in $K$ such that $t_{n} \in U_{n}$. Also choose $h_{n} \in C(K)$ with $0 \leq h_{n} \leq 1, h_{n}\left(t_{n}\right)=1$ and $h_{n}=0$ on $K \backslash U_{n}$. Put $g_{n}=1-h_{n}$. Clearly $\left\|g_{n}\right\| \leq 1$ and $g_{n}(t) \rightarrow 1$ for each $t \in K$.

Now $g_{n} f \in W C(K, X)_{1}$ and $g_{n}(t) f(t) \rightarrow f(t)$ for each $t \in K$. (For a future use in case (iii) we note here that until now the hypothesis of case (i) has not been used.)

Now if $\wedge$ is an extreme point of $W C(K, X)_{1}^{*}$, in view of the theorem quoted above, there exists a $t_{0} \in K$ such that

$$
\wedge\left(g_{n} f\right)=g_{n}\left(t_{0}\right) \wedge(f) \rightarrow \wedge(f) .
$$

Therefore $g_{n} f \rightarrow f$ in the weak topology.

However $\left\|g_{n} f-f\right\|=\left\|h_{n} f\right\| \geq\left\|f\left(t_{n}\right)\right\|$.

Since $\left\{f\left(t_{n}\right)\right\}$ converges weakly to a non-zero limit, we conclude that $g_{n} f \nrightarrow f$ in the norm.

Case (ii). Suppose $f(K)$ is an infinite set with 0 as the only accumulation point.

As before let $t_{n} \in K$ and $f\left(t_{n}\right) \rightarrow 0$ weakly. If $\left\|f\left(t_{n}\right)\right\| \nrightarrow 0$ the arguments as given in case (i) yield the required result.

So we assume w.l.o.g that $\left\|f\left(t_{n}\right)\right\| \rightarrow 0$.

Let $0<\delta<1$ and $N$ be such that $\left\|f\left(t_{n}\right)\right\|<\delta$ for all $n \geq N$.

Let $U_{n}=f^{-1}\left(f\left(t_{n}\right)\right)$. Then $U_{n}$ 's are clopen and pairwise disjoint.

Let $x_{0} \in X,\left\|x_{0}\right\|=1-\delta$. By $\tilde{x}_{0}$ we denote the constant function $x_{0}$ in $W C(K, X)$.

Let $f_{n}=\chi_{U_{n}} \tilde{x}_{0}$ for $n \geq N$. Clearly $f_{n}(t) \rightarrow 0 \forall t \in K$ and $\left\|f_{n}\right\|=\left\|x_{0}\right\|=1-\delta$.

Therefore $\left(f_{n}+f\right)(t) \rightarrow f(t)$ for all $t \in K$. Thus $f_{n}+f \rightarrow f$ weakly but not in norm. Note that for $t \in K \backslash U_{n}, f_{n}(t)=0$ and for $t \in U_{n},\left\|f_{n}(t)+f(t)\right\|=$ $\left\|x_{0}+f\left(t_{n}\right)\right\| \leq(1-\delta)+\delta=1$.

Hence the claim.

Case (iii). Suppose $f(K)$ is a finite set. Let $t_{0} \in K$ be such that $f\left(t_{0}\right) \neq 0$. Let $U=f^{-1}\left(f\left(t_{0}\right)\right)$. Suppose the clopen set $U$ is infinite. Get a sequence $\left\{g_{n}\right\}$ in $C(K)$ as in case (i) such that $g_{n}=0$ on $K \backslash U$ and $g_{n} \rightarrow \chi_{U}$ pointwise. Now $g_{n} f+\chi_{K \backslash U} f \rightarrow f$ pointwise and thus weakly.

Also,

$$
\begin{aligned}
\left\|g_{n} f+\chi_{K \backslash U} f\right\| & =\max \left\{\|f\|_{U},\|f\|_{K \backslash U}\right\} \\
& =\|f\|=1
\end{aligned}
$$


and

$$
\begin{aligned}
\left\|g_{n} f+\chi_{K \backslash U} f-f\right\| & \\
& =\left\|g_{n} f+\chi_{U} f\right\| \\
& =\left\|h_{n} f\right\|_{U}\left(\text { recall from case (i) that } g_{n}=1-h_{n}\right) \text { on } U \\
& =\left\|f\left(t_{0}\right)\right\| .
\end{aligned}
$$

Therefore $g_{n} f+\chi_{K \backslash U} f \nrightarrow f$ in the norm. The other situations are similarly handled.

Therefore $f$ is not a point of weak-norm continuity in $W C(K, X)_{1}$.

For any Banach spaces $X, Y$ let $\mathcal{L}(X, Y)$ denote the space of bounded operators.

Remark 1. For a dual space $X^{*}$, the space $W C\left(K, X^{*}\right)$ can be identified with $\mathcal{F}(X, C(K))$ (space of weakly compact operators; see [DS], page 490). When $X$ is reflexive, clearly $\mathcal{F}(X, C(K))=\mathcal{L}(X, C(K))$. Hence, when $K$ is infinite these spaces do not have denting points in their unit ball. If $(\Omega, \mathcal{A}, \mu)$ is an infinite measure space, this author has remarked in [R2] that the space $\mathcal{F}\left(L^{1}(\mu), X\right)$ is isometric to $W C(K, X)$ for an infinite compact space $K$. Therefore there are no denting points in the unit ball of $\mathcal{F}\left(L^{1}(\mu), X\right)$.

Corollary 1. For an infinite compact set $K$ and for any separable Banach space $X$, there are no points of weak-norm continuity in $\mathcal{L}(X, C(K))_{1}$.

Proof. It is well-known that the space $\mathcal{L}(X, C(K))$ can be identified with the space of $X^{*}$ valued functions on $K$ that are continuous when $X^{*}$ has the weak*topology (denoted as $W^{*} C\left(K, X^{*}\right)$; see [DS] page 490). The arguments given during the proof of Theorem 1 in [R1] can be used to show that any extreme point of $\mathcal{L}(X, C(K))_{1}^{*}$ is a point functional. Now the conclusion is obtained by proceeding along the same lines as in the proof of the above theorem. While in case (i), since $X$ is separable, note that $f(K)$ is a weak*-compact metric space.

If one merely wants to show that there are no denting points, then a shorter argument can be given using Theorem 1 of [S].

Corollary 2. For an infinite compact set $K$ and for any Banach space $X$, there are no denting points in $\mathcal{L}(X, C(K))_{1}$.

Proof. Let $T$ be a denting point in $\mathcal{L}(X, C(K))_{1}$. Since $T$ is in particular an extreme point, we have from Theorem 1 of $[\mathrm{S}]$, that $T^{*}$ attains its norm on an infinite set. Thus, as in case (i) of the above proof, a sequence can be constructed to converge weakly but not in the norm to $T$.

Remark 2. The identification of $\mathcal{L}\left(\ell^{1}\right)$ as $\mathcal{L}\left(c_{0}, C(\beta(N))\right)$ and that of $\mathcal{L}\left(\ell^{\infty}\right)$ as $\mathcal{L}\left(\ell^{\infty}, C(\beta(N))\right)$ shows that there are no denting points in their respective unit balls.

Remark 3. If $W$ is a closed subspace of $\mathcal{L}(X, C(K))$ containing $C\left(K, X^{*}\right)$ and is a $C(K)$ module, then using arguments similar to the ones given above one can show that there are no denting points in the unit ball of W. An application of this idea gives that there are no denting points in $\mathcal{L}\left(c_{0}\right)_{1}$ and in $\mathcal{L}\left(L^{1}(\mu)\right)_{1}$.

Remark 4. Recall that a point $x_{0}$ of $X_{1}$ is said to be strongly exposed if there exists an $x_{0}^{*} \in X^{*}$ such that $x_{0}^{*}\left(x_{0}\right)>x_{0}^{*}(x)$ for all other $x \in X_{1}$ and for any sequence $\left\{x_{n}\right\}$ in $X_{1}, x_{0}^{*}\left(x_{n}\right) \rightarrow x_{0}^{*}\left(x_{0}\right)$ implies $\left\|x_{n}-x_{0}\right\| \rightarrow 0$. Thus a strongly exposed point 
is in particular a denting point. Therefore our results give easy proofs of parts of Theorem 2 and Theorem 3 from $[\mathrm{G}]$ where it is shown that for an infinite $K$ and for any Hilbert space $H$ there are no strongly exposed points in $C(K, \mathcal{L}(H))_{1}$.

Even though we do not yet know the nature of extreme points of $W^{*} C(K, \mathcal{L}(H))_{1}$, the next proposition exhibits exposed points.

Proposition. Every exposed point of $C(K, \mathcal{L}(H))_{1}$ is an exposed point of $W^{*} C(K, \mathcal{L}(H))_{1}$.

Proof. Let $T$ be an exposed point of the unit ball of $C(K, \mathcal{L}(H))_{1}$. It follows from Theorem 2 in [G] that $H$ is separable and $K$ carries a strictly positive measure, say $\mu$. Now define a linear functional $\psi$ on $W^{*} C(K, \mathcal{L}(H))$ as in the proof of first part of Theorem 2 in [G]. Then the arguments given in [G] allow us to conclude that $\psi$ exposes $T$.

We conclude this note by applying these ideas in deciding the denting point question for the unit ball of injective tensor product spaces.

Theorem 3. Let $X$ be any Banach space and $Y$ an infinite dimensional $L^{1}$ predual space. There are no denting points in $\left(Y \otimes_{\epsilon} X\right)_{1}$.

Proof. Since $Y$ is an infinite dimensional space, clearly $Y^{* *}$ is isometric to an infinite dimensional $C(K)$ space. Suppose $x_{0}$ is a denting point of $\left(Y \otimes_{\epsilon} X\right)_{1}$. It is well known that $x_{0}$ is a denting point of $\left(Y \otimes_{\epsilon} X\right)_{1}^{* *}$. It follows from an observation in [E] that $Y^{* *} \otimes_{\epsilon} X$ is, under the canonical embedding, a subspace of $\left(Y \otimes_{\epsilon} X\right)^{* *}$. It is clear that $x_{0}$ continues to be a point of weak-norm continuity for the identity mapping on $\left(Y^{* *} \otimes_{\epsilon} X\right)_{1}$. Since $Y^{* *} \otimes_{\epsilon} X$ is isometric to $C(K, X)$, we get a contradiction. Hence the result.

\section{ACKnowledgement}

I thank Professor K. P. S. B. Rao for his helpful suggestions during the preparation of this note.

\section{Note ADDED ON DECEMBER 1, 1997}

The results of Benyamini, Rudin and Wage (Continuous images of weakly compact subsets of Banach spaces, Pacific J. Math. 70 (1977), 309-324) show that if $K$ is an infinite Eberlein compact space or $X$ is a subspace of a weakly compactly generated Banach space, then again accumulation points of $f(K)$ can be approximated by sequences and the arguments of this note work to show that there are no points of weak-norm continuity in $\mathcal{L}(X, C(K))_{1}$. In a recent work (Denting and strongly extreme points in the unit ball of spaces of operators) this author has shown that $\mathcal{L}\left(L^{1}(\mu), X\right)_{1}$ has a point of weak-norm continuity iff $L^{1}(\mu)$ is finite dimensional and $X_{1}$ has a point of weak-norm continuity. Also for any infinite, totally disconnected compact set $K$, there is no point of weak-norm continuity in $\mathcal{L}(X, C(K))_{1}$. Here however we could only exhibit nets that converge weakly but not in the norm. The general question is still open.

Added in proof. Theorem 2 was also proved using different methods by $\mathrm{Z}$. Hu and M. A. Smith in their paper On the extremal structure of the unit balls of Banach spaces of weakly continuous functions and their duals, that has appeared in Trans. Amer. Math. Soc. 349 (1997), 1901-1918. 


\section{REFERENCES}

[De] J.A. De Reyna, J. Diestel, V. Lomonosov and L.R. Piazza, Some observations about the space of weakly continuous functions from a compact space into a Banach space, Questiones Mathematicae 15 (1992), 415-425. MR 94b:46055

[DU] J. Diestel and J.J. Uhl, Vector measures, Math. Surveys, No. 15, Providence, Rhode Island, 1977. MR 56:12216

[D] J. Diestel, Sequences and Series in Banach spaces, GTM, No. 92, Springer, Berlin, 1984. MR 85i: 46020

[DS] N. Dunford and J.T. Schwartz, Linear operators, Part I: General Theory, Interscience publishers, New York, 1958. MR 90g:47001a (Reprint)

[E] G. Emmanuele, Remarks on weak compactness of operators defined on certain injective tensor products, Proc. Amer. Math. Soc., 116 (1992), 473-476. MR 92m:46109

[G] R. Grąślewicz, Extreme operator valued continuous maps, Arkiv for Matematik 29 (1991), 73-81. MR 92h:46049

[H] R.B. Holmes, Geometric functional analysis and its applications, GTM, No. 24, Springer, Berlin, 1975. MR 53:14085

[LLT] Bor-Luh Lin, Pei-Kee Lin and S.L. Troyanski, Characterizations of denting points, Proc. Amer. Math. Soc. 102 (1988), 526-528. MR 89e:46016

[L] H.E. Lacey, The isometric theory of classical Banach spaces, Springer, Berlin, 1974. MR 58: 12308

[R1] T.S.S.R.K. Rao, A note on extreme points of $W C(K, X)_{1}^{*}$, J. Ramanujan Math. Soc. 9 (1994), 215-219. MR 95j:46039

[R2] T.S.S.R.K. Rao, On a theorem of Dunford, Pettis and Phillips, Real Analysis Exchange 20 (1994/5), 741-743. MR 96d:46057

[S] M. Sharir, Characterization and properties of extreme operators into $C(Y)$, Israel J. Math. 12 (1972) 174-183. MR 47:5574

Indian Statistical Institute, R.V. College Post, Bangalore 560 059, India

E-mail address: tss@isibang.ac.in 